\title{
Apigenin suppresses the growth of colorectal cancer xenografts via phosphorylation and up-regulated FADD expression
}

\author{
QI RUI WANG ${ }^{1 *}$, XUE QING YAO ${ }^{2 *}$, GE WEN $^{3 *}$, QIN FAN $^{1}$, YING-JIA LI ${ }^{3}$, \\ XIU QIONG FU ${ }^{1}$, CHANG KE $\mathrm{LI}^{4}$ and XUE GANG SUN ${ }^{1}$ \\ ${ }^{1}$ The Key Laboratory of Molecular Biology, State Administration of Traditional Chinese Medicine, \\ School of Traditional Chinese Medicine, Southern Medical University; ${ }^{2}$ Guangdong General Hospital, \\ Guangdong Academy of Medical Sciences; ${ }^{3}$ Nan Fang Hospital; ${ }^{4}$ Yue Bei People's Hospital, Guangdong, P.R. China
}

Received August 4, 2010; Accepted October 18, 2010

DOI: $10.3892 / 01.2010 .215$

\begin{abstract}
Apigenin is a flavonoid belonging to the flavone structural class. It has been implicated as a chemopreventive agent against prostate and breast cancers. However, to the best of our knowledge, no published data are available regarding apigenin in colorectal cancer (CRC). The effects and mechanisms of apigenin on CRC may vary significantly. This study aimed to analyze the effects of apigenin on the growth of CRC xenografts in nude mice derived from SW480, as well as to investigate the underlying mechanisms. Whole-body fluorescence imaging is an inexpensive optical system used to visualize gene expression in small mammals using reporter genes, such as eGFP as a reporter. In our study, the expression of eGFP may reflect the size of the tumor. A terminal deoxynucleotidyl transferase dUTP nick end-labeling (TUNEL) assay showed that apigenin promoted the apoptosis of CRC cells. Furthermore, the expression of five genes related to the proliferation and apoptosis of CRC, i.e., cyclin D1, BAG-1, $\mathrm{Bcl}-2$, yrdC and Fas-associated protein with death domain (FADD), was detected by real-time quantitative RT-PCR. Among these genes, the up-regulated expression of FADD was noted in CRC xenograft tumors treated with apigenin. Immunohistochemistry and Western blotting confirmed the results at the protein level. Furthermore, Western blot analysis
\end{abstract}

Correspondence to: Dr Xue Gang Sun, The Key Laboratory of Molecular Biology, State Administration of Traditional Chinese Medicine, School of Traditional Chinese Medicine, Southern Medical University, No. 1838, Guang Zhou Da Dao Bei Road, Guangzhou, Guangdong 510515, P.R. China

E-mail:sxg_smu@126.com

Dr Chang Ke Li, Department of Anesthesiology, Yue Bei People's Hospital, Shaoguan, Guangdong 512026, P.R. China

E-mail: anesthesia@126.com

*Contributed equally

Key words: apigenin, colorectal cancer, whole-body fluorescence imaging system, Fas-associating protein with death domain showed that apigenin induced the phosphorylation of FADD. Our findings suggest that apigenin enhances the expression of FADD and induces its phosphorylation, which may cause apoptosis of CRC cells and inhibition of tumor growth.

\section{Introduction}

Colorectal cancer (CRC) is one of the leading causes of morbidity and mortality both in the United States and worldwide. In 2009, the estimated new cases of colon and rectum cancers were 106,100 and an estimated 49,920 patients succumbed in the United States alone (1). Approximately 850,000 people develop CRC annually and 500,000 succumb to the disease (2). The prevention and treatment of CRC thus remains a global health issue.

Apigenin is a flavonoid belonging to the flavone structural class and is chemically known as 4',5,7,-trihydroxyflavone (3). Apigenin has been shown to possess significant antiinflammatory, antioxidant and anti-carcinogenic properties (4). Pre-treatment with apigenin induces the apoptosis of HCT-116 colon cancer cells (5) and inhibits the growth of colon carcinoma cell lines, including SW480, HT-29 and Caco-2 (6). A previous study showed that apigenin enhanced apoptosis in HT29 cells with wild-type adenomatous polyposis coli (7). In vivo studies showed that apigenin moderately reduces azoxymethane-induced colon carcinogenesis and inhibits the growth of colorectal cancer (8). However, to the best of our knowledge no published data are currently available regarding the in vivo study of apigenin on the growth of CRC in mice. Therefore, an in vivo study using fluorescence imaging may aid in a detailed analysis of the effectiveness of apigenin on xenograft tumors derived from SW480, a cell line established from a primary adenocarcinoma of the colon (9).

\section{Materials and methods}

Cell culture. SW480/enhanced green fluorescent protein (eGFP) was donated by Dr Zhao, Department of Pathology, Southern Medical University, China. Tumor cells were cultured in RPMI-1640 medium (Gibco, Grand Island, NY, USA) supplemented with $10 \%$ fetal bovine serum (Hyclone, Logan, UT, USA), $100 \mathrm{U} / \mathrm{ml}$ penicillin/streptomycin (Gibco) 
Table I. Primer sequence information.

Gene name

Primers

Product (bp)

\begin{tabular}{ll}
\hline BAG-1 & Sense 5' - ACTGTCACCCACAGCAATGA-3' \\
& Antisense 5' - TGTGGAACCCCTATGACCTC-3' \\
Bcl-2 & Sense 5' - ATGTGTGTGGAGAGCGTCAA-3' \\
& Antisense 5' - ACAGTTCCACAAAGGCATCC-3' \\
CCND1 & Sense 5' - GATCAAGTGTGACCCGGACT-3' \\
& Antisense 5' - TCCTCCTCTTCCTCCTCCTC-3' \\
FADD & Sense 5' - CCTGTGTGCAGCATTTAACG-3' \\
& Antisense 5' - GTCCTCGATGCTGTCGATCT-3' \\
yrdC & Sense 5' - CGGATTCCTGATCATGCTTT-3' \\
& Antisense 5' - AGGACAACTGAGGCCAGAGA-3' \\
GAPDH & Sense 5' - ACAGTCAGCCGCATCTTCTT -3' \\
& Antisense 5' - ACGACCAAATCCGTTGACTC -3' \\
\hline
\end{tabular}

and $500 \mu \mathrm{g} / \mathrm{ml} \mathrm{G} 418$, and incubated in a humidified chamber with $5 \% \mathrm{CO}_{2}$ at $37^{\circ} \mathrm{C}$.

Mice and xenograft tumors. Male BALB/c-nu mice (specific pathogen-free) were obtained from the Laboratory Animal Center of Southern Medical University. The animals were maintained at a constant room temperature with a 12-h light cycle in a conventional animal colony. The mice were between 6 and 8 weeks old at the beginning of the experiments, and weighed 15-20 g. Tumor xenografts were generated by subcutaneous injection of $5 \times 10^{6}$ cells on the right inguen of the nude mice. The procedures were performed using approved protocols, in accordance with the guidelines of the Animal Care and Use Committee of Southern Medical University. Three days following the injection, the fluorescence emitted by the tumor cells was imaged by a whole-body GFP imaging system (Imagestation 2000MM; Kodak, USA) to visualize the formation of the tumor. A total of 16 tumor-bearing mice were randomly divided into two groups, the apigenin and control groups. Apigenin (SigmaAldrich, USA) was dissolved in dimethyl sulfoxide (DMSO) and diluted in phosphate-buffered saline (PBS). Each mouse in the apigenin group was injected intraperitoneally with 20 $\mathrm{mg} / \mathrm{kg}$ of apigenin, while in the control group, each mouse was injected with the same amount of vehicle solution (DMSO-PBS). Images of tumors were obtained every 6 days and analyzed to calculate the tumor area and tumor growth using Molecular Imaging Software Version 4.0 provided by Kodak 2000MM System.

Terminal deoxynucleotidyl transferase dUTP nick endlabeling (TUNEL) assay. A TUNEL apoptosis assay kit for paraffin section was purchased from Nanjing KeyGen Biotech. Inc., China. The procedures were performed in accordance with the manufacturer's instructions. Through microscopic observation, cells were defined as apoptotic if the nuclei were positively stained. The positive cells were counted in three high-power fields. The apoptotic index (AI) was calculated as the percentage of positively-stained cells: $\mathrm{AI}=$ (number of positive cells/total number of nucleated cells) x $100 \%$.
Real-time quantative RT-PCR. The primer sequences are shown in Table I. cDNA was synthesized by oligo dT primed reverse transcription from $2 \mu \mathrm{g}$ of total RNA using an access RT system (Takara). Real-time PCR was performed using the Mx3005P real-time PCR system (Stratagene) and Stratagene's Brilliant SYBR-Green QPCR Master Mix kit, following the manufacturer's instructions. Thermal cycling conditions were: $95^{\circ} \mathrm{C}$ for $5 \mathrm{~min}$ and 40 cycles at $95^{\circ} \mathrm{C}$ for $10 \mathrm{sec}$, followed by $60^{\circ} \mathrm{C}$ for $30 \mathrm{sec}$. Human glyceraldehyde-3-phosphate dehydrogenase (GAPDH) gene was amplified as an internal control. The target and GAPDH genes were amplified in the same reaction. The relative quantification was determined using the $2^{-\Delta \Delta \mathrm{Ct}}$ method.

Immunohistochemistry assay. The tissues were embedded and cut into $4-\mu \mathrm{m}$ sections. Immunohistochemistry was performed according to the protocol as previously described (10). The sections were incubated with the primary antibodies [rabbit anti-Fas-associated protein with death domain (FADD) antibody, Cell signaling technology (CST)] and biotinylated secondary antibody (Zymed Laboratories Inc., South San Francisco, CA, USA). Nuclear counterstaining was performed using haematoxylin. Brown stains in the immunostained regions was considered to be FADD-positive staining. The scoring approach in the assessment of immunohistochemically-stained tissue sections was based on a simple and reproducible protocol as previously described (10).

Western blotting. The protein lysates were loaded onto $10 \%$ SDS-polyacrylamide gel, electrotransferred to polyvinylidene fluoride (PVDF) (Immobilon P; Millipore, Bedford, MA, USA) membranes and blocked with $5 \%$ non-fat dry milk in Tris-buffered saline (TBST, $100 \mathrm{mM} \mathrm{NaCl}, 50 \mathrm{mM}$ Tris, $0.1 \%$ Tween-20; $\mathrm{pH} 7.5)$. The membranes were incubated with FADD, anti-phospho-FADD (Ser 194) (CST) polyclonal antibodies or anti-GAPDH monoclonal antibody (CST) overnight at $4^{\circ} \mathrm{C}$, respectively, followed by $1-\mathrm{h}$ incubation with horseradish peroxidase (HRP)-conjugated secondary antibody. The signal was detected using an enhanced chemiluminescence system (ECL; CST) and documented with a charge-coupled 
A

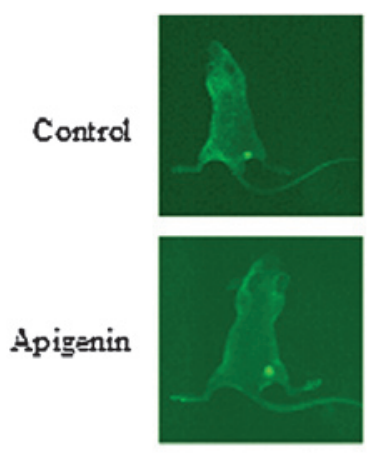

$3 d$
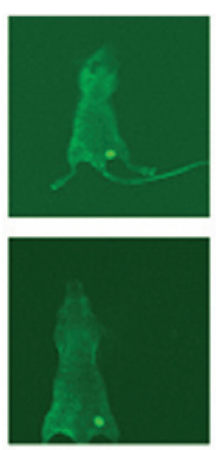

$9 d$
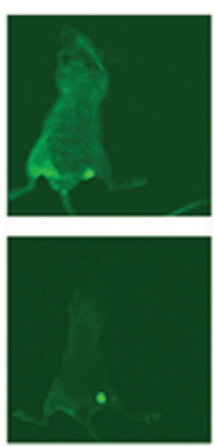

$15 d$
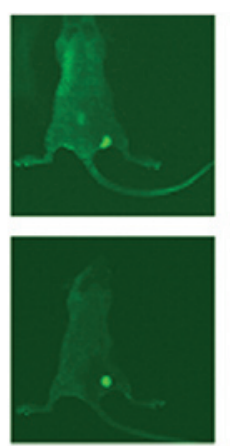

$21 d$
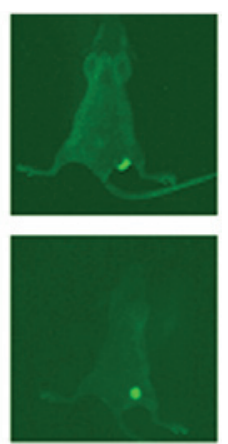

$27 d$

B

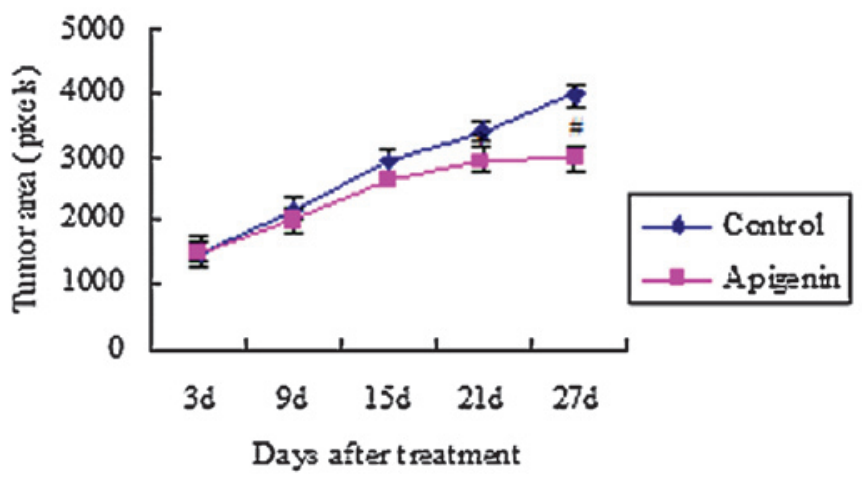

Figure 1. Effects of apigenin on the growth of xenograft colorectal tumors. (A) Representative images in vivo of xenograft tumors with stably-expressed GFP. (B) Effects of apigenin on tumor. Apigenin significantly decreased the growth of tumors compared to the control group. ${ }^{\#} \mathrm{P}<0.01$, * $\mathrm{P}<0.05 \mathrm{vs}$. control group.

device system (Imagestation 2000MM; Kodak). Data analysis was performed using the Molecular Imaging Software Version 4.0. The protein level in each sample was normalized to the level of GAPDH for the corresponding sample.

Statistical analysis. The experiments were repeated a minimum of three times. The data were expressed as means $\pm \mathrm{SD}$. The data were analyzed with the Statistical Package (version 13.0; SPSS). Differences between the two groups were analyzed using Student's t-test. $\mathrm{P}<0.05$ was considered to be statistically significant.

\section{Results}

Effects of apigenin on the growth of tumor xenografts. The tumor areas increased rapidly from 1525.88 to 3991.38 pixels in the control group, while the tumor areas increased from 1496.13 to 2963.38 pixels in the apigenin group after 27 days. Apigenin treatment inhibited the growth of colorectal cancer xenografts after $21(\mathrm{P}<0.05)$ and 27 days $(\mathrm{P}<0.01)$ compared to the control group (Fig. 1).

Effects of apigenin on the apoptosis of tumor and expression of $F A D D$. Based on TUNEL staining, a higher number of brownstained cells were found in the apigenin group compared to those of the control group $(\mathrm{P}<0.01)$ (Fig. 2A-C). Five genes that related to the proliferation and apoptosis of CRC, i.e., cyclin D1 (CCND1), BAG-1, Bcl-2, yrdC and FADD were screened, and it was found that FADD was up-regulated by apigenin (Fig. 2D).
Immunological staining showed that the expression of FADD was mainly present in the cytoplasm of CRC cells in the control group, while the overexpression of FADD was observed in both the cytoplasm and nucleus (Fig. 2E-F) in the apigenin pre-treated mice. Western blotting showed that the phosphorylation $(\mathrm{P}<0.05)$ and expression $(\mathrm{P}<0.05)$ of FADD increased significantly in the apigenin group compared to the control group (Fig. 2G-H).

\section{Discussion}

The real-time in vivo imaging system utilizes light emitted by a bioluminescent or fluorescent reporter gene (or fluorescent molecule, such as a dye or quantum dot) expressed in a living organism and analyzes the source and strength of that bioluminescent or fluorescent signal non-invasively, allowing for extensive longitudinal modeling in the same live animal (11). In our study, a CRC cell line stably expressing eGFP was selected since cancer growth is observed directly in live animals by epifluorescence. A high correlation was found between the fluorescence intensity of the cancer and the mass of cancer cells. Regarding CRC tumors, an imaging system would be more reliable for sizing, due to the difficulty experienced in compressing the CRC tumor with the caliper (12). Our study using in vivo imaging shows that apigenin decreases the region of colon cancer derived from SW480 by increasing AI. Apigenin inhibits the proliferation of the human anaplastic thyroid carcinoma cell line (ARO). The inhibitory effect is associated with the increase of programmed cell 


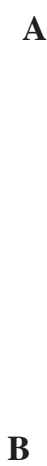

A

B
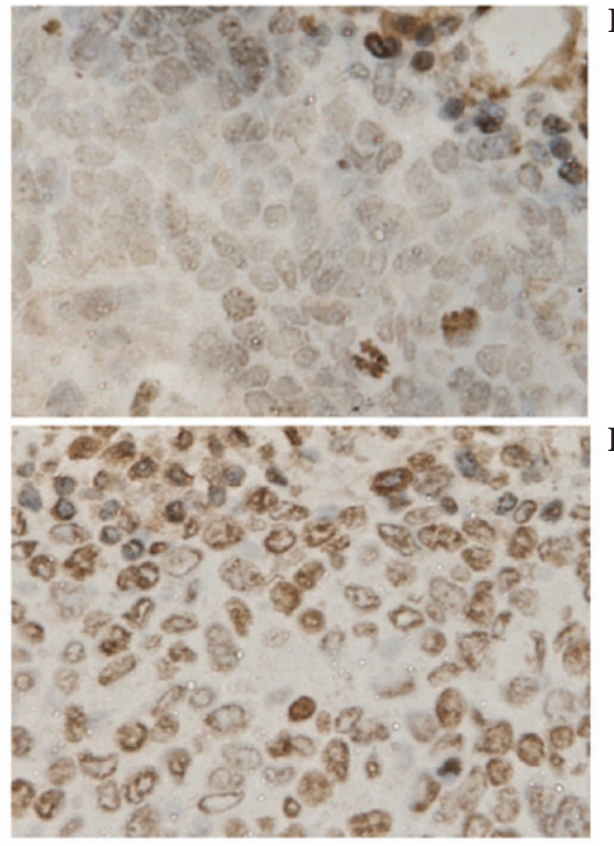

C

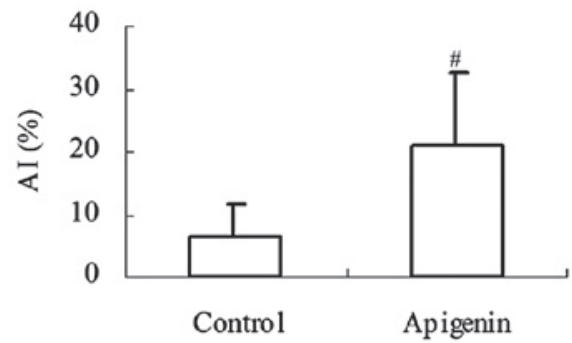

D

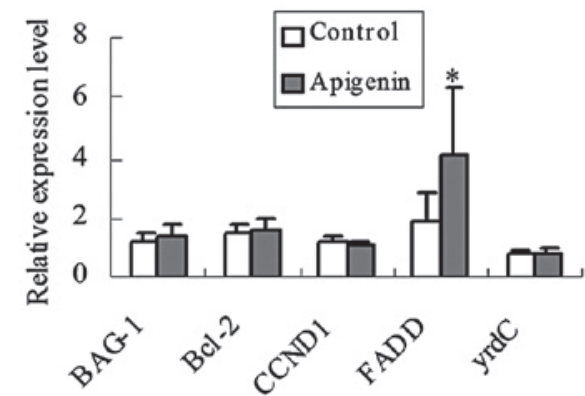

$\mathbf{E}$

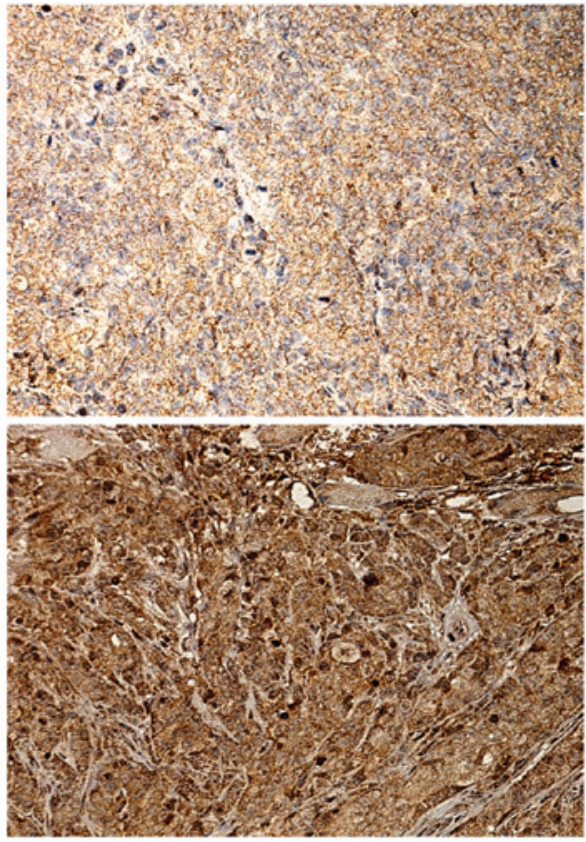

G

Control Apigenin

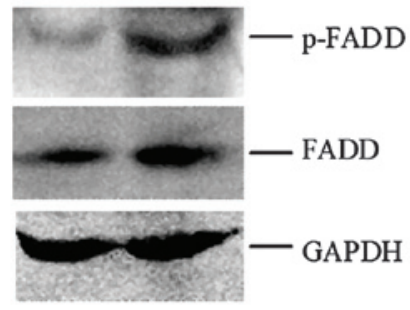

H

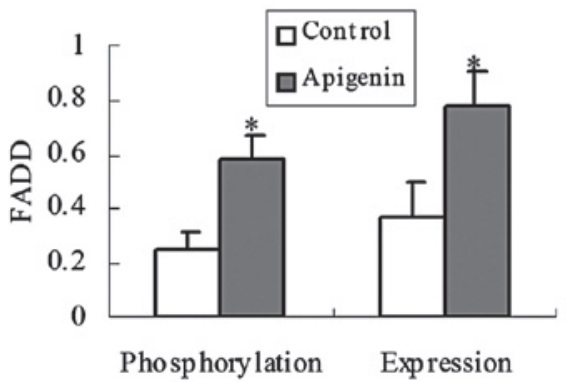

Figure 2. Effects of apigenin on the apoptosis of tumor and expression of FADD. (A-C) TUNEL analysis of apoptotic cells in colorectal tumor. Apoptosis index (C) shows that apigenin (B) increased the number of apoptotic cells compared to the control group (A). (D) Real-time RT-PCR evaluates the expression of CCND1, BAG-1, Bcl-2, yrdC and FADD. Apigenin significantly up-regulated the expression of FADD compared to the control group. (E-F) Immunohistochemical assay detected FADD expression in the tumor. Apigenin significantly increased the expression of FADD (F) compared to the control group (E). (G-H) Western blotting detected the expression and phosphorylation of FADD in the tumor (G). A semi-quantative analysis showed that apigenin increases the expression and phosphorylation of FADD compared to the control group. ${ }^{~} \mathrm{P}<0.01,{ }^{*} \mathrm{P}<0.05$ vs. control group.

death (13). Treatment with apigenin significantly inhibited the cell proliferation in MDA-MB-453 cells that were resistant to 5-fluorouracil. This inhibition resulted in apoptosis, as evidenced by the increased number of apoptotic cells and the activation of caspase-3 (14). Taken together, the results suggest that apigenin inhibits the proliferation of cancer cells by up-regulating the cell apoptosis rate.

To establish why apigenin inhibits the growth of CRC, five genes were selected to determine the effect of apigenin on the proliferation and apoptosis of CRC. CCND1 (15) and BAG-1 (16) overexpression in colorectal tumor cells contribute to abnormal growth and tumorigenicity, and the expression of yrdC promotes the proliferation of SW-480 (17). A decreased expression of $\mathrm{Bcl}-2$ in tumor cells significantly relates to increased tumor size (18). Transfection of a dominant negative FADD prevents the induction of apoptosis (19). Therefore, the five genes were screened by real-time quantative RT-PCR and only FADD was found to be up-regulated by apigenin.

Fas is a death receptor, transducing cell death signaling upon stimulation by Fas ligand. During Fas-initiated cell death signaling, the formation of Fas-death-inducing signaling complex (Fas-DISC) is the initial step. Previous observations 
showed that FADD is an essential component of DISC, which contains a death domain and induces apoptosis when overexpressed in multiple cell types (20-23). Our results show that FADD expression is up-regulated in CRC cells with a high $\mathrm{AI}$, which is consistent with the observation that transfection of a dominant-negative mutant of FADD decreases the apoptotic response of SW480 cells to resveratrol (24). These results suggest that cells reprogrammed to increase the abundance of FADD may promote apoptosis in cells doomed to die. Since quantitatively phosphorylated FADD plays an essential role in Burkitt lymphoma BJAB cell cycle arrest at the $\mathrm{G} 2 / \mathrm{M}$ phase (25), the up-regulated phosphorylation of FADD induced by apigenin may be involved in the regulation of proliferation of CRC cells. Thus, we propose that apigenin suppresses the growth of CRC by enhancing the expression of and inducing the phosphorylation of FADD, which mediates the apoptosis of tumor cells and inhibits cell proliferation. However, further studies should be conducted as to which mechanisms of phosphorylation and translocation of FADD to nucleus are involved in order to elucidate the preventive effects of apigenin on the growth of CRC.

\section{Acknowledgements}

This study was supported by grants from the National Natural Sciences Foundation of China (No. 30670580).

\section{References}

1. Jemal A, Siegel R, Ward E, Hao Y, Xu J and Thun MJ: Cancer statistics. CA Cancer J Clin 59: 225-249, 2009.

2. Benson AB III: Epidemiology, disease progression, and economic burden of colorectal cancer. J Manag Care Pharm 13: S5-S18, 2007.

3. Noh HJ, Sung EG, Kim JY, Lee TJ and Song IH: Suppression of phorbol-12-myristate-13-acetate-induced tumor cell invasion by apigenin via the inhibition of p38 mitogen-activated protein kinase-dependent matrix metalloproteinase-9 expression. Oncol Rep 24: 277-283, 2010.

4. Patel D, Shukla S and Gupta S: Apigenin and cancer chemoprevention: Progress, potential and promise (review). Int J Oncol 30: 233-245, 2007.

5. Farah M, Parhar K, Moussavi M, Eivemark S and Salh B: 5,6-Dichloro-ribifuranosylbenzimidazole- and apigenin-induced sensitization of colon cancer cells to TNF-alpha-mediated apoptosis. Am J Physiol Gastrointest Liver Physiol 285: G919-G928, 2003.

6. Wang W, Heideman L, Chung CS, Pelling JC, Koehler KJ and Birt DF: Cell-cycle arrest at G2/M and growth inhibition by apigenin in human colon carcinoma cell lines. Mol Carcinog 28 102-110, 2000.

7. Chung CS, Jiang Y, Cheng D and Birt DF: Impact of adenomatous polyposis coli (APC) tumor supressor gene in human colon cancer cell lines on cell cycle arrest by apigenin. Mol Carcinog 46: 773-782, 2007.

8. Au A, Li B, Wang W, Roy H, Koehler K and Birt D: Effect of dietary apigenin on colonic ornithine decarboxylase activity, aberrant crypt foci formation, and tumorigenesis in different experimental models. Nutr Cancer 54: 243-251, 2006.

9. Leibovitz A, Stinson JC, McCombs WB III, McCoy CE, Mazur KC and Mabry ND: Classification of human colorectal adenocarcinoma cell lines. Cancer Res 36: 4562-4569, 1976.
10. Zhao L, Wang H, Li J, Liu Y and Ding Y: Overexpression of Rho GDP-dissociation inhibitor alpha is associated with tumor progression and poor prognosis of colorectal cancer. J Proteome Res 7: 3994-4003, 2008.

11. Liu L, Zhang QL, Jiang HY and Ding YQ: Establishment of a whole-body visualization model of orthotopically implanted colorectal carcinoma and metastasis model in nude mice. Nan Fang Yi Ke Da Xue Xue Bao 27: 1161-1163, 1166, 2007.

12. Cemazar M, Golzio M, Escoffre JM, Couderc B, Sersa G and Teissié J: In vivo imaging of tumor growth after electrochemotherapy with cisplatin. Biochem Biophys Res Commun 348: 997-1002, 2006.

13. Yin F, Giuliano AE and van Herle AJ: Signal pathways involved in apigenin inhibition of growth and induction of apoptosis of human anaplastic thyroid cancer cells (ARO). Anticancer Res 19: 4297-4303, 1999.

14. Choi EJ and Kim GH: 5-Fluorouracil combined with apigenin enhances anticancer activity through induction of apoptosis in human breast cancer MDA-MB-453 cells. Oncol Rep 22: 1533-1537, 2009.

15. Arber N, Doki Y, Han EK, Sgambato A, Zhou P, Kim NH, Delohery T, Klein MG, Holt PR and Weinstein IB: Antisense to cyclin D1 inhibits the growth and tumorigenicity of human colon cancer cells. Cancer Res 57: 1569-1574, 1997.

16. Barnes JD, Arhel NJ, Lee SS, Sharp A, Al-Okail M, Packham G, Hague A, Paraskeva C and Williams AC: Nuclear BAG-1 expression inhibits apoptosis in colorectal adenoma-derived epithelial cells. Apoptosis 10: 301-311, 2005.

17. Tao GQ, Yang M, Jiang HH, Yan YQ and Wang XH: The experiment on expression of human yrdC promotes proliferation of colon cancer SW-480 cells, Shanghai Med J 31: 596-598, 2008.

18. Ofner D, Riehemann K, Maier H, Riedmann B, Nehoda H, Tötsch M, Böcker W, Jasani B and Schmid KW: Immunohistochemically detectable bcl-2 expression in colorectal carcinoma: correlation with tumor stage and patient survival. $\mathrm{Br}$ J Cancer 72: 981-985, 1995

19. Chinnaiyan AM, Tepper CG, Seldin MF, O'Rourke K, Kischkel FC, Hellbardt S, Krammer PH, Peter ME and Dixit VM: FADD/MORT1 is a common mediator of CD95 (Fas/ APO-1) and tumor necrosis factor receptor-induced apoptosis. J Biol Chem 271: 4961-4965, 1996.

20. Boldin MP, Varfolomeev EE, Pancer Z, Mett IL, Camonis JH and Wallach D: A novel protein that interacts with the death domain of Fas/APO1 contains a sequence motif related to the death domain. J Biol Chem 270: 7795-7798, 1995.

21. Chinnaiyan AM, O'Rourke K, Tewari M and Dixit VM: FADD, a novel death domain-containing protein, interacts with the death domain of Fas and initiates apoptosis. Cell 81: 505-512, 1995.

22. Yanase N, Kanetaka $Y$ and Mizuguchi J: Interferon- $\alpha$-induced apoptosis via tumor necrosis factor-related apoptosis-inducing ligand (TRAIL)-dependent and -independent manner. Oncol Rep 18: 1031-1038, 2007.

23. Lu HF, Lai KC, Hsu SC, Lin HJ, Yang MD, Chen YL, Fan MJ, Yang JS, Cheng PY, Kuo CL and Chung JG: Curcumin induces apoptosis through FAS and FADD, in caspase-3-dependent and -independent pathways in the N18 mouse-rat hybrid retina ganglion cells. Oncol Rep 22: 97-104, 2009.

24. Delmas D, Rébé C, Lacour S, Filomenko R, Athias A, Gambert P, Cherkaoui-Malki M, Jannin B, Dubrez-Daloz L, Latruffe N and Solary E: Resveratrol-induced apoptosis is associated with Fas redistribution in the rafts and the formation of a death-inducing signaling complex in colon cancer cells. J Biol Chem 278: 41482-41490, 2003.

25. Scaffidi C, Volkland J, Blomberg I, Hoffmann I, Krammer PH and Peter ME: Phosphorylation of FADD/MORT1 at serine 194 and association with a 70-kDa cell cycle-regulated protein kinase. J Immunol 164: 1236-1242, 2000. 